\title{
No solar hiding place for greenhouse sceptics
}

A study has confirmed that there are no grounds to blame the Sun for recent global warming. The analysis shows that global warming since 1985 has been caused neither by an increase in solar radiation nor by a decrease in the flux of galactic cosmic rays (M. Lockwood and C. Fröhlich Proc. R. Soc. A doi:10.1098/rspa.2007.1880; 2007). Some researchers had suggested that the latter might influence global warming through an involvement in cloud formation.

"This paper is the final nail in the coffin for people who would like to make the Sun responsible for present global warming," says Stefan Rahmstorf, a climate scientist at the Potsdam Institute for Climate Impact Research in Germany.

Claims that the Sun, rather than raised levels of greenhouse gases, has been responsible for recent warming have persisted in a small number of scientists and in parts of the media. Mike Lockwood, a physicist at the Rutherford Appleton Laboratory in Chilton, UK, says he was "galvanized" to carry out the comprehensive study by misleading media reports. $\mathrm{He}$ cites 'The Great Global Warming Swindle', a television programme shown in March by Britain's Channel 4, as a prime example.

Together with Claus Fröhlich of the World Radiation Center in Davos, Switzerland, Lock- wood brought together solar data for the past 100 years. The two researchers averaged out the 11-year solar cycles and looked for correlation between solar variation and global mean temperatures. Solar activity peaked between 1985 and 1987. Since then, trends in solar irradiance, sunspot number and cosmic-ray intensity have all been in the opposite direction to that required to explain global warming.

In 1997, Henrik Svensmark, a physicist at the Danish National Space Center in Copenhagen, suggested that cosmic rays facilitate cloud formation by seeding the atmosphere with trails of ions that can help water droplets form (H. Svensmark and E. J. Friis-Christensen J. Atmos. Solar-Terrest. Phys. 59, 1225-1232; 1997). He proposed that, as a result of this, changes in the Sun's magnetic field that influence the flux of cosmic rays could affect Earth's climate. This led to claims that cosmic rays are the main influence on modern climate change.

Even in the face of the new analysis, Svensmark insists that solar theories should not be dismissed. "If you look at temperatures in the troposphere, there is a remarkable correlation with solar activity," he says. Lockwood insists that none of the tropospheric data show the trend that the solar theory would need.

Nir Shaviv, an astrophysicist at the Hebrew University of Jerusalem who has championed

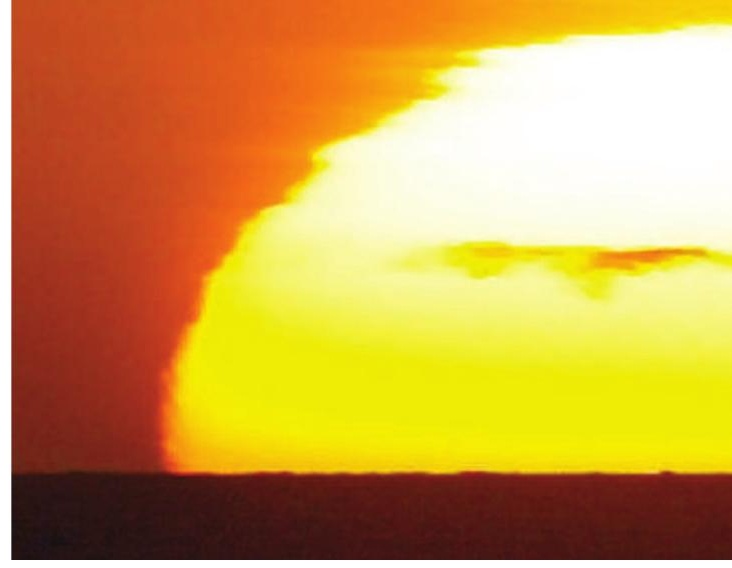

Hot topic: solar activity peaked too early to have caused the current trends in climate change.

a Sun-climate link, argues that there may be a lag in Earth's reaction to the Sun because of the thermal inertia of the oceans.

But other climate researchers find the idea of a 'hidden' time lag unconvincing. "With each year, and with each new set of data that comes in, a time lag becomes ever more unlikely," says Urs Neu, deputy head of ProClim-, the climate and global change forum of the Swiss Academy of Sciences in Bern.

On other timescales, however, Sun-climate links may remain worthy of study. "Climate change is a cocktail of many effects," says Jasper Kirkby, a physicist at CERN, the European

\section{Cosmic-ray results auger well for future}

The first substantial results from the world's largest detector of cosmic rays are being presented this week at a conference in Mexico.

The Pierre Auger Cosmic Ray Observatory is already the largest cosmic-ray observatory in the world even though it is only threequarters finished. It sprawls across more than 3,000 square kilometres of the Argentine pampas near the city of Malargüe - an area a tenth the size of Belgium. Researchers from the 15 countries collaborating on the US\$50-million project hope that after its 1,600

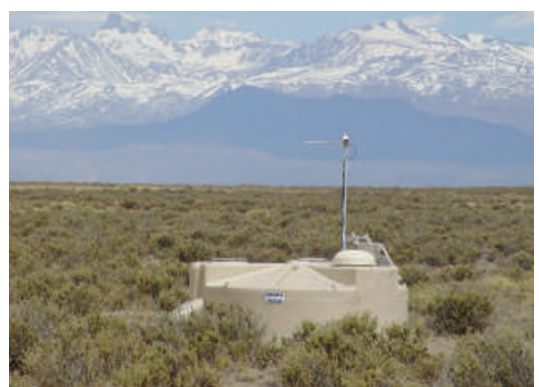

A small component of a large observatory.

detectors and 24 telescopes have been commissioned around the end of this year the observatory may reveal the origins of the highest-energy particles bombarding Earth from space.

Among the results being presented at the International Cosmic Ray Conference in Merída, Yucatán on 3-11 July are new measurements of the energy spectrum of cosmic rays. The team confirms that above a threshold energy known as the GZK cut-off the number of cosmic-ray events falls sharply. Particles that start off with energies above this threshold are expected to be slowed down by interactions with the cosmic microwave background as they travel. Cosmic rays significantly above the cutoff energies would thus have to come from relatively nearby, which would imply that they were produced by strange new physics, such as the spontaneous decay of unknown massive particles.

An experiment in Japan called the Akeno Giant Air Shower Array sparked excitement when it failed to see the cut-off in data obtained up to 2004. Since then, though, data from the High Resolution Fly's Eye - a detector in Utah - have 


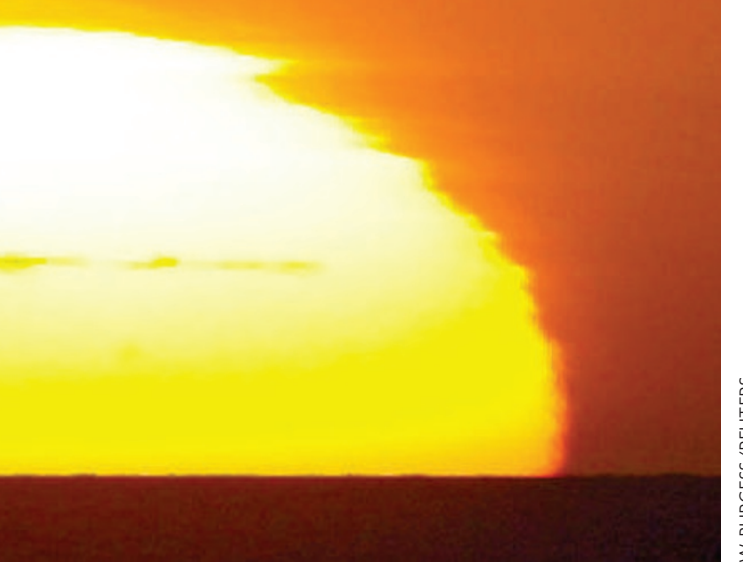

particle-physics laboratory near Geneva in Switzerland, who is leading an experiment aimed at simulating the effect of cosmic rays on clouds. "Past climate changes have clearly been associated with solar activity. Even if this is not the case now, it is still important to understand how solar variability affects climate."

Ken Carslaw, an atmospheric scientist at the University of Leeds, UK, points out that solar effects might still be possible. They might have acted to cool the climate in recent decades, but been overwhelmed. If so, the climate could be more sensitive to greenhouse gases than is generally thought, and future temperature increases might be greater than expected if a countervailing solar effect comes to an end. Quirin Schiermeier

provided evidence for the reality of the cut-off. The new Pierre Auger data seem to confirm that the cut-off is real.

If there is no new physics in the form of particle decays or other exotic effects, though, there should be plenty of new astrophysics in understanding what sort of celestial mechanism might be pumping up incoming protons to energies millions of times higher than those produced by the most powerful Earth-based accelerators. One crucial step is to identify where in the sky they are coming from. Alan Watson of the University of Leeds, UK, a spokesman for the Pierre Auger project, says that the team has identified some interesting candidate regions of the sky, but won't reveal the details until it has obtained more data.

“Unless I talk in my sleep, even my wife doesn't know what these regions are," he says. Jenny Hogan

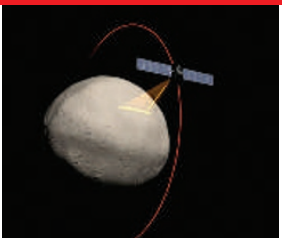

ASTEROID MISSION GEARS UP FOR LAUNCH

NASA mission leaders hope Dawn will rise on time.

www.nature.com/news

\section{The great gig in the South}

When Al Gore and his colleagues dreamed up the idea of a climate-change consciousness-raising Live Earth event featuring concerts on every continent, they came up against a problem. The event's 7 July date corresponds with midwinter in the Antarctic, when no one gets on or off the seventh continent. So flying in Bono and Madonna to frolic with penguins would not be an option.

Enter Nunatak, a five-strong band of guitar, bass, drums, fiddle and sax, whose members are part of the crew at the Rothera Research Station, run by the British Antarctic Survey (BAS).

It is not the only band on the continent. Every New Year's Day, the US McMurdo base hosts the Icestock festival, and even in winter there's plenty of music. "All the bases have bands except maybe Bird Island, as there are only four overwintering people there," explains Julius Rix at the BAS's more southerly Halley base. But being the farthest north of the bigger bases gives Nunatak an advantage. "Rothera has double the satellite bandwidth compared to us," says Rix. And so Nunatak got the gig.

Even so, the webcam set-up on Rothera isn't quite up to sending broadcast-quality sound and visuals live to the world. "To do it live we would need a very fast satellite link," says Matt Balmer, an electronics engineer and Nunatak's guitarist and songwriter. So the performance will be recorded 'as live' a day or so beforehand. "We're effectively emailing it in," says Balmer.

Despite the technical delay, the performance should be pretty authentic. The band plans to perform outside - which might make their instruments "sound a bit funny", according to the BAS's Linda Capper, back in balmy Cambridge, $\mathrm{UK}$, not withstanding the rigours faced by the performers.

Balmer is tough enough to dismiss such worries: "It's only minus six outside; you get used to these sorts of temperatures."

Nor does the prospect of a global audience chill him, in part because it doesn't feel that real. "We don't have an appreciation of it," says Balmer. "We see the world through the Internet." The last ship out of Rothera left in April. "Until October we won't see anybody else."

Saxophonist and marine biologist Alison Massey expects the rest of the base to be an appreciative audience, not least because after weeks of rehearsal at close quarters, "it will be the last time they'll hear the songs". Balmer hopes for something a bit more rock and roll: "There will probably be groupies throwing their underwear at us," he says. With only 17 people in the audience, though, matching the knickers to the punters may not be too hard.

Katharine Sanderson

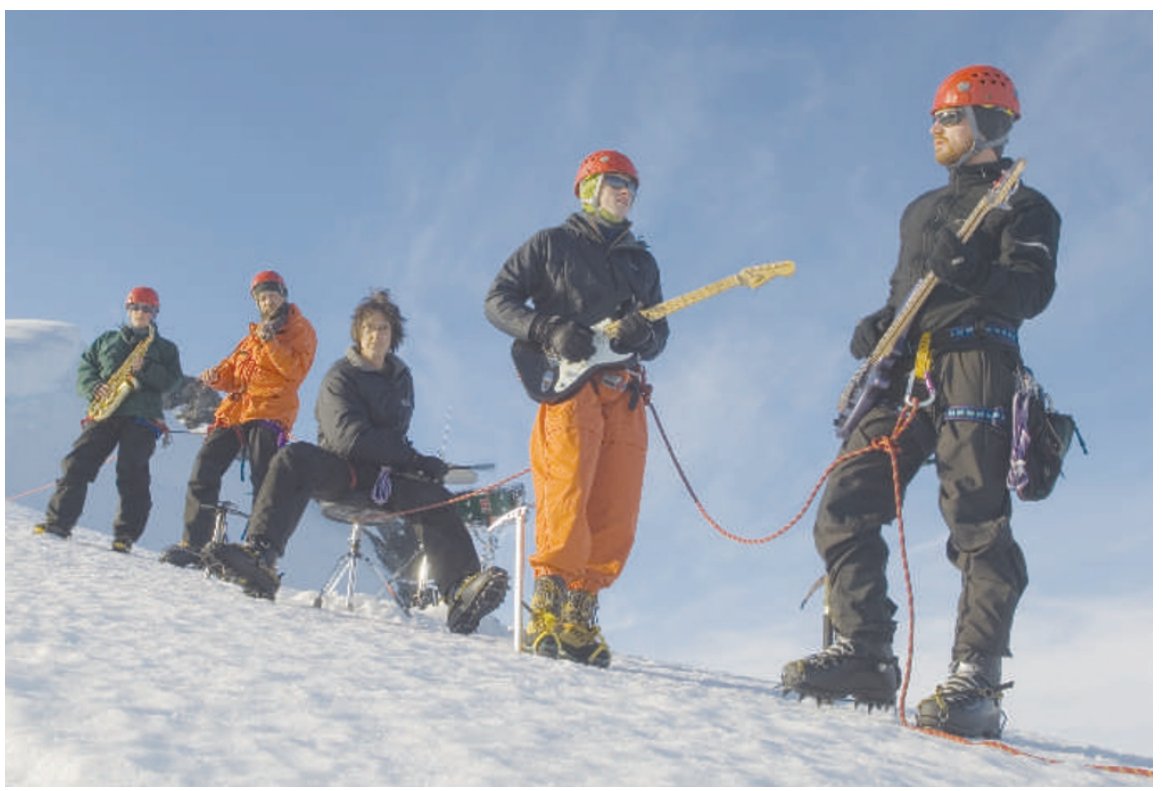

Antarctic brass monkeys: Nunatak will perform in sub-zero temperatures for Live Earth. 